\title{
Corrigendum
}

Jeffrey A. Wilhite*, Khemraj Hardowar, Harriet Fisher, Barbara Porter, Andrew B. Wallach, Lisa Altshuler, Kathleen Hanley, Sondra R. Zabar and Colleen C. Gillespie

\section{Clinical problem solving and social determinants of health: a descriptive study using unannounced standardized patients to directly observe how resident physicians respond to social determinants of health}

https://doi.org/10.1515/dx-2020-0125

Published online October 27, 2020

Corrigendum to: Jeffrey A. Wilhite*, Khemraj Hardowar, Harriet Fisher, Barbara Porter, Andrew B. Wallach, Lisa Altshuler, Kathleen Hanley, Sondra R. Zabar and Colleen C. Gillespie. Clinical problem solving and social determinants of health: a descriptive study using unannounced standardized patients to directly observe how resident physicians respond to social determinants of health. Diagnosis 2020, Volume 7, Issue 3, pages 313-324. https://doi.org/10. 1515/dx-2020-0002.
*Corresponding author: Jeffrey A. Wilhite*, MPH, Department of Medicine, Division of General Internal Medicine and Clinical Innovation, NYU School of Medicine, 462 1st Avenue, CD629, New York, NY, 10016, USA, Phone: +1 646501 4120, E-mail: Jeffrey.Wilhite@nyulangone.org. https://orcid.org/00000003-4096-8473

Khemraj Hardowar, Harriet Fisher, Barbara Porter, Andrew B. Wallach, Lisa Altshuler, Kathleen Hanley and Sondra R. Zabar, Department of Medicine, Division of General Internal Medicine and Clinical Innovation, NYU School of Medicine, New York, NY, USA Colleen C. Gillespie, Department of Medicine, Division of General Internal Medicine and Clinical Innovation, NYU School of Medicine, New York, NY, USA; and Institute for Innovations in Medical Education, NYU School of Medicine, New York, NY, USA
Unfortunately, a typographic error in the results portion of the abstract was missed during final stages of proofing and editing. The count of full elicitors should read as $38 / 68$ rather than $28 / 68$, and the $\%$ of negative elicitors is $23 \%$. The corrected results read as follows: Residents fell into three groups when it came to clinical problem-solving around a housing trigger for asthma: those who failed to ask about housing and therefore did not uncover mold as a potential trigger (neglectors - 21\%; 14/68); those who asked about housing in negative ways that prevented disclosure and response (negative elicitors - 23\%, 16/68); and those who elicited and explored the mold issue (full elicitors $56 \%$; 38/68). 